\title{
The Adoption of Mobile Health Applications by Patients in Developing Countries: A Systematic Review
}

\author{
Nasser Aljohani ${ }^{1}$, Daniel Chandran ${ }^{2}$ \\ Faculty of Engineering and Information Technology \\ University of Technology Sydney Australia, Sydney, Australia
}

\begin{abstract}
Mobile health (m-health) apps adoption in developing countries is a new research area in the healthcare industry. M-health is comparatively recent in information systems, with little attention being paid to it developing countries in the previous years. Applications of the $m$-health strategies in developing nations are considered one of the best platforms for guaranteeing the citizenry's safety and healthcare security. A systematic review was conducted of $\mathrm{m}$-health apps adoption by patients in developing countries to evaluate the current results. It reviews 22 papers that were published on the topic of $\mathbf{m}$-health adoption in developing countries in academic journals and conferences over the last decade. It identifies the research in terms of research methodologies, theories and models adopted, significant factors identified, limitations and recommendations. Findings show there is a limited contribution to $m$-health apps adoption in developing countries. Most studies employed TAM and focused on the technological and individual levels; very low intention has been made to health-related factors, levels, and theories. The review presents a broad overview of previous academic studies with a view to future research.
\end{abstract}

Keywords-M-health; mobile health; apps; adoption; review; developing countries

\section{INTRODUCTION}

Mobile technology has grown in use in the healthcare delivery and health results in developed countries in the last few years. Electronic health (e-health) refers to computer-based services, while mobile health (m-health) applies to mobile systems with specialized features to improve health care delivery [5]. The concept "m-health" was first used by Prof. Isteparian, who refers to mobile devices and networking systems used for healthcare delivery [28]. Fig. 1 presents the role of both e-health and m-health.

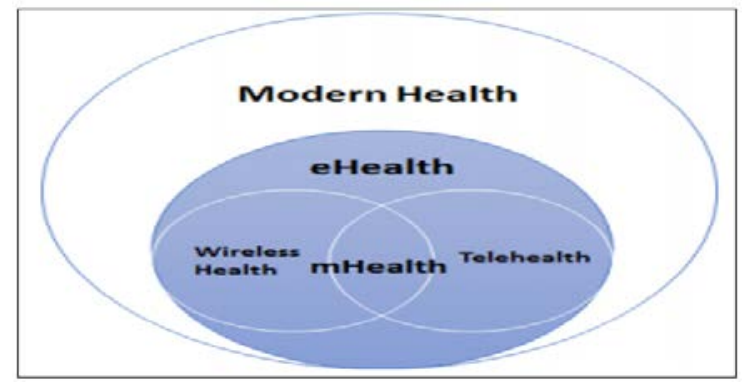

Fig. 1. Role of E-health and M-health (Adopted from [26]).
In Fig. 2, Dehzad et al. classify m-health into three classes of technologies: devices, sensors, and applications [10]. Besides, they categorized the target group of m-health into healthy people, hospital patients, and chronically ill individuals. M-health solutions can carry four areas: wellness and prevention, diagnosis, treatment and [10]. There are currently more than 165,000 health apps available on smartphone online stores [9]. According to Larson [29], mhealth apps categorized by searchers into four different types:

- Information app: Provide general health information to the public.

- Diagnostic app: To enter patients' information and provide a diagnosis to physicians.

- Control app: Assist medical devices with remote monitoring and control.

- Adapter app: Transform smartphones to become mobile medical devices.

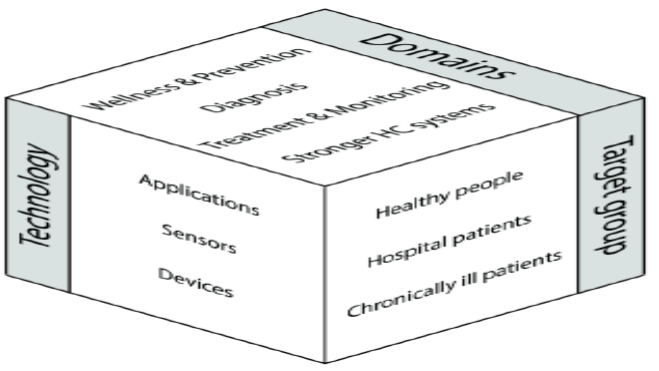

Fig. 2. Classification Model for M-health Solutions (Adopted from [10]).

Having m-health technology will promote healthcare awareness and access to knowledge that will enhance the lives of the citizenry and provide the developed countries with the opportunity to create a sustainable workforce with economic resilience [16]. The majority of mobile phone users, especially smartphones and computing technology in developing nations, have adopted mobile devices to access information, particularly about the right healthcare facilities that offer quality services to guarantee security and healthcare safety. The initiative of downloading mobile apps for understanding the proper treatment procedures and the most appropriate types of medication has enabled the majority of the citizens in the developing nations to access Medicare conveniently and reduce cost [1]. The adoption of m-health gives the governments of 
the developing nations the advantage to guarantee patients safety and health information records by considering data protection and visualization strategies. Some developing countries, such as Malaysia, Thailand, China, and India, have been introducing technological advances in healthcare systems to improve the treatment process [24].

Despite the prospective benefits of m-health apps, adopting and accepting such a technology is not as widespread as expected in developing countries [30]. Factors found to be influencing m-health have been explored in several studies. However, an insufficient contribution has been paid to the factors affecting the adoption of m-health by patients; besides, the factors influencing e-health adoption rates in developed countries have been comprehensively reviewed against mhealth. In the sense of developing countries, we conducted a systematic literature review to properly comprehend and verify the adoption of m-health apps. This is an especially significant discovery in the advancement of a modern research area. It provides a possibility to step back and review several samples, methods, and theories collected from different studies in mhealth. Thus, this research seeks to play a key role in enhancing the research in this rapidly growing field of m-health. Beyond that to examine the existing state of m-health among people of devolving countries.

\section{LiTERATURE SEARCH APPROACH}

Several keyword sets were evaluated to have a sufficiently reliable and authentic secondary source. For instance, the sets of keywords considered for the study include "adoption of mhealth", "adoption of mobile health", and "adoption of mhealth" and this focused on the use and application of Google Scholar as one of the most reliable search engines for academic journals with both comprehensive and conclusive information about the initiative of developing the m-health in the developing nations. The technique that was idealized to establish the search setting considered extensive and varied studies published between 2010 and 2020. The core underlying principle for choosing the wide range of timeline is to present information with a broad historical background about the nature of the developing countries' approach to adopting mhealth.

The major reasoning for choosing the wide range of timelines is dependent on two fundamental reasons. First, due to the nature and the quality of healthcare outcomes before and after the developing nations begun the initiative of adopting the $\mathrm{m}$-health. It is imperative to note that restricting the healthcare systems in developing countries by adopting the m-health strategy has improved and stands a better chance to improve the citizens' overall quality of lifestyle in the developing nations in the foreseeable future [25]. Second, by 2013, the use and application of mobile technology to access and receive healthcare services had become most popular since both the government and the citizenry in the developing nations considered the strategy one of the best platforms of accessing quality healthcare conveniently and at a reduced cost [14]. The use of mobile apps can be a fast and reliable way to provide healthcare to large numbers of people who cannot make a physical visit to a healthcare facility.

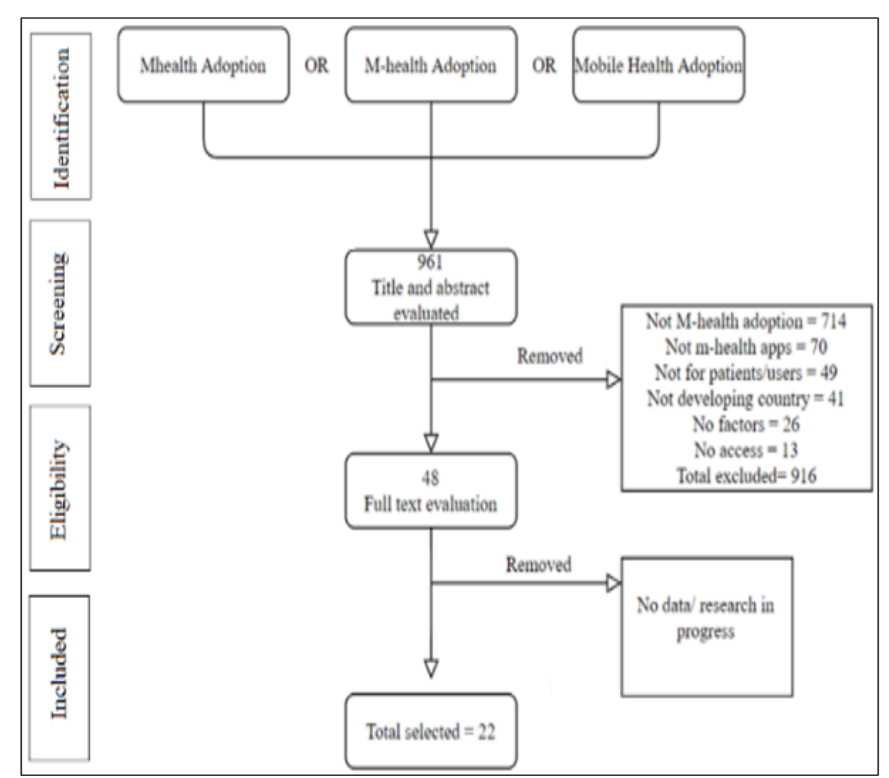

Fig. 3. Flowchart of the Search Strategy.

A total of 22 secondary sources were selected for the study, particularly academic journals, to investigate the approach taken by the developing nations for reasons of adopting the $\mathrm{m}$ health strategies. These studies proved relevant and authentic about affirming the perspective that the developing countries should adopt the m-health approach to improve the overall quality of lifestyle and health condition of their citizens. Using the PRISMA flow diagram [32], Fig. 3 shows the research selection strategy.

\section{LITERATURE ANALYSIS}

This section explains several of the existing primary literature concerning m-health from a scholarly perspective. The analysis included the date of publication, theories and methods used the identified significant factors, and the limitations and findings of the m-health applications research.

\section{A. General Analysis}

1) Publication date analysis: Publication dates were analyzed to determine the latest most significant developments in research publications. As shown in Fig. 4, the number of articles increased from 2012 until 2015. Then, the number increased to reach 3 to 4 papers during the years 2016 to 2018. No complete studies have been conducted in developing countries during the last two years. This could be because mhealth concept is new or has not been applied thoroughly in developing countries.

2) Countries and number of participants analysis: The analysis of studies conducted in developing countries showed that ten studies were conducted in China, five studies in Bangladesh, two in Jordan, one in Arab countries, one in Taiwan, one in Malaysia, and one in United Arab Countries. Table I and Fig. 5 show broad information about each study. 


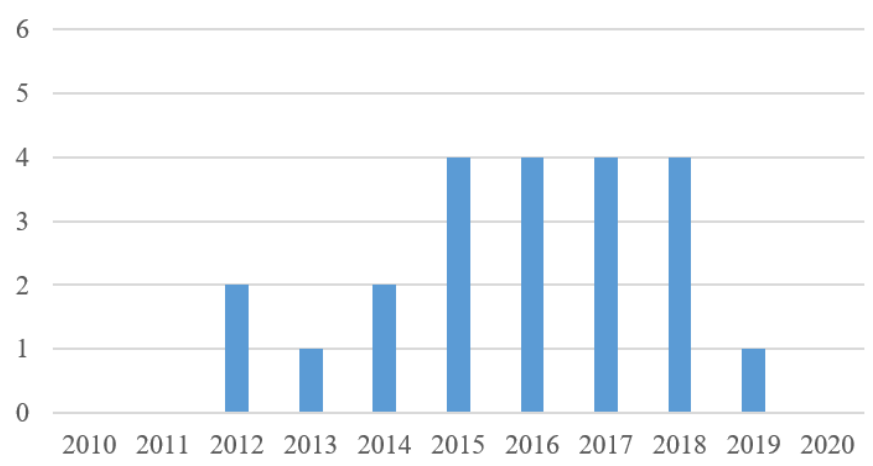

Fig. 4. Number of Studies Per Years (2010-2020).

TABLE I. DETAILS OF THE STUDIES

\begin{tabular}{|c|c|c|c|}
\hline Country & Targeted group & Participants & Reference \\
\hline Arab countries & $\begin{array}{l}\text { Elderly Arab m- } \\
\text { health users }\end{array}$ & 134 & [7] \\
\hline Bangladesh & $\begin{array}{l}\text { Young citizen of } \\
\text { public and private } \\
\text { universities }\end{array}$ & 144 & {$[20]$} \\
\hline Bangladesh & Elderly users & 375 & [14] \\
\hline Bangladesh & All & 227 & {$[21]$} \\
\hline Bangladesh & Patients & 37 & [27] \\
\hline Bangladesh & $\begin{array}{l}\text { Elderly users above } \\
60\end{array}$ & 274 & {$[22]$} \\
\hline Bangladesh & All & 296 & [3] \\
\hline China & All & 429 & [17] \\
\hline China & Elderly users & 212 & [33] \\
\hline China & $\begin{array}{l}\text { M-health users } \\
\text { Over } 40\end{array}$ & 424 & [11] \\
\hline China & All & 481 & [35] \\
\hline China & M-health users & 428 & [18] \\
\hline China & All & 650 & [17] \\
\hline China & $\begin{array}{l}\text { Hypertensive } \\
\text { patients }\end{array}$ & 157 & [13] \\
\hline China & $\begin{array}{l}\text { M-health service } \\
\text { users }\end{array}$ & 650 & {$[36]$} \\
\hline China & All & 388 & [12] \\
\hline China & $\begin{array}{l}\text { Elderly users above } \\
60\end{array}$ & 395 & [31] \\
\hline Jordan & All & 366 & [15] \\
\hline Jordan & All & 365 & {$[2]$} \\
\hline Malaysia & All & 480 & [24] \\
\hline Taiwan & Young users & 170 & [23] \\
\hline $\begin{array}{l}\text { United Arab } \\
\text { Emiratis }\end{array}$ & M-health users & 144 & {$[6]$} \\
\hline
\end{tabular}

\section{B. Theories and Models Used}

Different theories and model have been used in the 22 studies. These include the Technology and Acceptance Model, Theory of Reasoned Action (TRA), Unified Theory of Acceptance and Use (UTAUT), Protection Motivation Theory (PMT), Theory of Planned Behavior (TPB). It has been noted that the TAM model is the most applied model among the 22 selected papers. Some researchers used the same model, extended, or even combined it with other models. Two studies proposed hypotheses without specifying any theory or model. In addition, two studies have suggested new models. However, one study, which applied quantitative methodology, did not involve any theory. Table II shows the theories used in each study and their references.

\section{Research Methodologies}

The most popular research approach used in the m-health area is the quantitative research technique, while qualitative research is used only by Khatun et al. [27] out of the 22 studies. This could be due to the impossibility to interview patients directly. Health conditions of patients could be the possible reason.

\section{Significant Factors}

This section identifies only factors that have been proven to be affecting the behavioral intention in different countries. It has been noted the most significant factor identified in most studies is the preserved ease of use. The following Table III shows the significant factors, the number of studies, and their references.

TABLE II. THEORIES AND MODELS USED

\begin{tabular}{|l|l|l|}
\hline $\begin{array}{l}\text { Theory/ } \\
\text { Model }\end{array}$ & $\begin{array}{l}\text { Number of } \\
\text { studies }\end{array}$ & Reference \\
\hline TAM & 10 & {$[6][7][13][17][20][21][33][15][23][36]$} \\
\hline UTAUT & 5 & {$[2][3][14][22][33]$} \\
\hline TRA & 2 & {$[33][35]$} \\
\hline PMT & 2 & {$[18][33]$} \\
\hline TPB & 2 & {$[33][11]$} \\
\hline New model & 2 & {$[17][31]$} \\
\hline No Model & 2 & {$[24][27]$} \\
\hline VAB & 1 & {$[11]$} \\
\hline
\end{tabular}


TABLE III. IDENTIFIED SIGNIFICANT FACTORS

\begin{tabular}{|c|c|c|c|c|c|}
\hline Factor & Reference & $\mathbf{N}$ & Factor & Reference & $\mathbf{N}$ \\
\hline \multicolumn{6}{|l|}{ Technical } \\
\hline Perceived ease of use & $\begin{array}{l}{[2][6][7][12][13][15][20][21]} \\
{[23][33]}\end{array}$ & 10 & Perceived usefulness & $\begin{array}{l}{[2][6][12][15][20][21][23]} \\
{[33]}\end{array}$ & 8 \\
\hline Performance expectancy & [3] [14] [22] [33] & 4 & Facilitating conditions & [3] [14] [35] & 3 \\
\hline Effort expectancy & [3] [14] [22] & 3 & Resistance to change & {$[13][22]$} & 2 \\
\hline Technology anxiety & {$[22]$} & 1 & Technological incapability & {$[27]$} & 1 \\
\hline Lack of access & [27] & 1 & Technological incapability & {$[27]$} & 1 \\
\hline \multicolumn{6}{|l|}{ Individual } \\
\hline Attitude & [7] [23] [24] [33] [35] & 6 & Trust & [6] [12] [15] [19] [27] [31] & 6 \\
\hline Age & [18] [19] [23] & 3 & Self-Efficacy & [13] [18] [33] & 3 \\
\hline Response Efficacy & {$[18][33]$} & 2 & Awareness & [2] [27] & 2 \\
\hline Gender differences & {$[27][35]$} & 2 & Innovativeness & {$[2]$} & 1 \\
\hline $\begin{array}{l}\text { Smartphone technology usage } \\
\text { experience }\end{array}$ & [13] & 1 & Illiteracy & [27] & 1 \\
\hline perceived reliability & {$[3]$} & 1 & Language & {$[27]$} & 1 \\
\hline Perceived Behavioral Control & [33] & 1 & Poverty & {$[27]$} & 1 \\
\hline Waiting time & [14] & 1 & Perceived personalization & [19] & 1 \\
\hline \multicolumn{6}{|l|}{ Social/Cultural/Environmental } \\
\hline Social influence & [2] [3] [13] [14] [15] [22] & 6 & Subjective norm & {$[21][33][35]$} & 3 \\
\hline Culture & {$[7]$} & 1 & & & \\
\hline \multicolumn{6}{|l|}{ Security/Privacy } \\
\hline Privacy & [12] [15] [19] & 3 & Security & [6] [15] & 2 \\
\hline Performance risks & {$[12]$} & 1 & & & \\
\hline \multicolumn{6}{|l|}{ Health } \\
\hline Perceived Severity & [18] [33] & 2 & Perceived Vulnerability & [18] [33] & 2 \\
\hline Relationship with the doctor & [13] & 1 & Support from hospital & {$[31]$} & 1 \\
\hline Chronic disease & {$[12]$} & 1 & $\begin{array}{l}\text { Declining physiological } \\
\text { conditions }\end{array}$ & {$[31]$} & 1 \\
\hline
\end{tabular}

\section{Discussion of RESUlTS AND Limitations}

The number of studies in m-health adoption in developing countries is still low compared to the total number of devolving nations. There is an increased interest and attention by researchers in China, 10 studies, and Bangladesh, 6 studies, about the adoption of m-health apps by patients in the last ten years. However, the number of studies comparing to the number of developing countries is still low. Previous literature analysis revealed that little to no attempt to exploit qualitative or mixed methods had been made by present researchers. Only one research out of the 22 papers has used the qualitative approach. The qualitative method in healthcare can answer difficult questions or questions that may not be answered by quantitative research considering the context in which it has been examined [4]. Since mixed study methodology affords a clearer perspective and further interpretations of the conclusions and can provide richer account of healthcare than any approach can provide alone [34], both qualitative and mixed methodologies are crucially needed in m-health studies.
Regarding theories and models applied to m-health adoption studies, the TAM model has been mostly used, followed by UTAUT over other theories. The selected papers mostly focused on the technological and individual levels with the minimal intention to health-related factors. M-health is a combination of technology and health areas. Only three studies have mentioned some health-related factors in their studies, the role of chronic disease [12], declining physiological conditions [31], and relationship with doctors [13]. Some studies have used the theories with no consideration of the context that had been examined. Meng et al. [31] state a limitation of their study that it may not be applicable to other countries due to cultural differences. Chandran and Aljohani state that Saudi Arabian culture, for example, is a mixture of both traditions and Islamic believes and call for more consideration [8]. This is also applicable to other Arab and Islamic nations. From the analysis of the selected 22 studies, only one study considered cultural affect. Hence, there is a need to put more efforts to examine health-related factors by considering cultural aspects. 
Age has been considered as a targeted group in some studies. For example, elderly m-health users have been included as the main targeted group in five studies [7-14-2233-31] and young m-health users in two studies [20-23]. It would be more useful for future contributions to consider age to be a moderator to target more participants instead of specifying the sample size. Only two studies have examined the role of gender as a significant factor. Hence, there is a need to put some efforts into age and gender as moderators of factors affecting the adoption of m-health apps in developing countries.

In summary, there is a call for more studies about m-health adoption in developing countries. Exploring the adoption of mhealth by applying qualitative or mixed methods will yield more excellent perspectives and offer more reliable evidence to $\mathrm{m}$-health apps studies. There is more space for future research to analyze the impact of m-health on health and cultural factors.

\section{CONCLUSION}

A systematic review of literature to evaluate at m-health app adoption in developing countries between 2010 and 2020 was conducted for this study. Among 48 studies, 22 studies were included in the review, thereby proving to be suitable. Most studies had used quantitative methodology, but this particular one chose the qualitative method, and no one attempted to employ a mixed method. As most studies used the TAM model and focused on the technological and individual levels, the very low intention has been made to other healthrelated factors, levels, and theories. Moreover, there is a lack to consider the culture being examined. The review presents a broad overview of previous academic studies with a view to future research. This study would be useful as guide to other researchers in the future. Lastly, another research field for ehealth is to look into whether e-health research can also be extended to m-health.

\section{REFERENCES}

[1] A. Alaiad, M. Alsharo, and Y. Alnsour, "The Determinants of M-Health Adoption in Developing Countries: An Empirical Investigation," Applied clinical informatics, vol. 10, no. 5, pp. 820-840, 2019.

[2] A. Alalwan, A. M. Baabdullah, N. P. Rana, Y. K. Dwivedi, F. Hudaib, and A. Shammout, "Examining the Factors Affecting Behavioural Intention to Adopt Mobile Health in Jordan," in Conference on eBusiness, e-Services and e-Society, Springer, pp. 459-467, 2018.

[3] M. Z. Alam, W. Hu, and Z. Barua, "Using the UTAUT model to determine factors affecting acceptance and use of mobile health (mHealth) services in Bangladesh," Journal of Studies in Social Sciences, vol. 17, no. 2, 2018.

[4] Z. Q. Al-Busaidi, "Qualitative research and its uses in health care," Sultan Qaboos University Medical Journal, vol. 8, no. 1, p. 11, 2008.

[5] N. Aljohani and D. Chandran, "Adoption of M-Health Applications: The Saudi Arabian Healthcare Perspectives," in Australasian Conference on Information Systems, Perth Western Australia, AIS eLibrary, pp. 180186, 2019.

[6] M. Alloghani, A. Hussain, D. Al-Jumeily, and O. Abuelma'atti, "Technology Acceptance Model for the Use of M-Health Services among health related users in UAE," in 2015 International Conference on Developments of E-Systems Engineering (DeSE), IEEE, pp. 213-217, 2015.

[7] A. Alsswey, I. Naufal, and B. Bervell, "Investigating the Acceptance of Mobile Health Application User Interface Cultural-Based Design to Assist Arab Elderly Users," International Journal of Advanced Computer
Science and Applications, vol. 9, doi: 10.14569/IJACSA.2018.090819, 2018.

[8] D. Chandran and N. Aljohani, "The Role of Cultural Factors on Mobile Health Adoption: The Case of Saudi Arabia," AMCIS 2020 Proceedings 3, 2020.

[9] C. Crico, C. Renzi, N. Graf, A. Buyx, H. Kondylakis, L. Koumakis, and G. Pravettoni, "mHealth and telemedicine apps: in search of a common regulation," ecancermedicalscience, vol. 12, 2018.

[10] F. Dehzad, C. Hilhorst, C. de Bie, and E. Claassen, "Adopting health apps, what's hindering doctors and patients?," Health, vol. 6, no. 16, p. 2204, 2014.

[11] Z. Deng, X. Mo, and S. Liu, "Comparison of the middle-aged and older users' adoption of mobile health services in China," International journal of medical informatics, vol. 83, no. 3, pp. 210-224, 2014.

[12] Z. Deng, Z. Hong, C. Ren, W. Zhang, and F. Xiang, "What predicts patients' adoption intention toward mHealth services in China: empirical study," JMIR mHealth and uHealth, vol. 6, no. 8, p. e172, 2018.

[13] K. Dou, P. Yu, N. Deng, F. Liu, Y. Guan, Z. Li, Y. Ji, N. Du, X. Lu, and H. Duan, "Patients' Acceptance of Smartphone Health Technology for Chronic Disease Management: A Theoretical Model and Empirical Test," JMIR mHealth and uHealth, vol. 5, no. 12, pp. e177, 2017.

[14] Y. K. Dwivedi, M. A. Shareef, A. C. Simintiras, B. Lal, and V. Weerakkody, "A generalised adoption model for services: A crosscountry comparison of mobile health (m-health)," Government Information Quarterly, vol. 33, no. 1, pp. 174-187, 2016.

[15] K. M. Faqih, and M.-I. R. M. Jaradat, "Mobile healthcare adoption among patients in a developing country environment: Exploring the influence of age and gender differences," International Business Research, vol. 8, no. 9, pp. 142, 2015.

[16] M.-P. Gagnon, P. Ngangue, J. Payne-Gagnon, and M. Desmartis, "mHealth adoption by healthcare professionals: a systematic review," Journal of the American Medical Informatics Association, vol. 23, no. 1, pp. 212-220, 2016.

[17] X. Guo, J. Yuan, X. Cao, and X. Chen, "Understanding the acceptance of mobile health services: A service participants analysis," in 2012 International Conference on Management Science \& Engineering 19th Annual Conference Proceedings, IEEE, pp. 1868-1873, 2012.

[18] X. Guo, X. Han, X. Zhang, Y. Dang, and C. Chen, "Investigating mhealth acceptance from a protection motivation theory perspective: gender and age differences," Telemedicine and e-Health, vol. 21, no. 8, pp. 661-669, 2015.

[19] X. Guo, X. Zhang, and Y. Sun, "The privacy-personalization paradox in mHealth services acceptance of different age groups," Electronic Commerce Research and Applications, vol. 16, pp. 55-65, 2016.

[20] M. R. Hoque, M. R. Karim, and M. B. Amin, "Factors affecting the adoption of mHealth services among young citizen: A Structural Equation Modeling (SEM) approach," Asian Business Review, vol. 5, no. 2, pp. 60-65, 2015.

[21] M. R. Hoque, "An empirical study of mHealth adoption in a developing country: the moderating effect of gender concern," BMC medical informatics and decision making, vol. 16, no. 1, p. 51, 2016.

[22] R. Hoque and G. Sorwar, "Understanding factors influencing the adoption of mHealth by the elderly: An extension of the UTAUT model," International journal of medical informatics, vol. 101, pp. 75-84, 2017.

[23] M.-C. Hung and W.-Y. Jen, "The adoption of mobile health management services: an empirical study," Journal of Medical Systems, vol. 36, no. 3, pp. 1381-1388, 2012.

[24] Z. Hussein, W. Oon, and A. Fikry, "Consumer Attitude: Does It Influencing the Intention to Use mHealth?," Procedia Computer Science, vol. 105, pp. 340-344, doi: 10.1016/j.procs.2017.01.231, 2017.

[25] B. Hwabamungu and Q. Williams, "m-Health adoption and sustainability prognosis from a care givers' and patients' perspective," in Proceedings of the 2010 Annual Research Conference of the South African Institute of Computer Scientists and Information Technologists, 2010, pp. 123-131.

[26] International Pharmaceutical Federation (IPF), Use of mobile health tools in pharmacy practice, May 1, 2019. Accessed on: December 20, 2020. [Online]. Available: https://www.fip.org/files/content/publications/2019/ mHealth-Use-of-mobile-health-tools-in-pharmacy-practice.pdf 
[27] F. Khatun, A. E. Heywood, P. K. Ray, A. Bhuiya, and S.-T. Liaw, "Community readiness for adopting mHealth in rural Bangladesh: a qualitative exploration," International journal of medical informatics, vol. 93, pp. 49-56, 2016.

[28] E. G. Kariuki and P. Okanda, "Adoption of m-health and usability challenges in m-health applications in Kenya: Case of Uzazi Poa mhealth prototype application," in 2017 IEEE AFRICON, IEEE, pp. 530535, 2017

[29] R. S. Larson, "A Path to Better-Quality mHealth Apps," JMIR mHealth and uHealth, vol. 6, no. 7, p. e10414, 2018.

[30] L. Lee and A. Sheikh, "Understanding stakeholder interests and perspectives in evaluations of health IT," Evidence-Based Health Informatics: Promoting Safety and Efficiency Through Scientific Methods and Ethical Policy, vol. 222, p. 53, 2016.

[31] F. Meng, X. Guo, Z. Peng, K.-H. Lai, and X. Zhao, "Investigating the Adoption of Mobile Health Services by Elderly Users: Trust Transfer Model and Survey Study," JMIR mHealth and uHealth, vol. 7, no. 1, p. e12269, 2019.
[32] Moher D, Liberati A, Tetzlaff J, Altman DG, The PRISMA Group (2009). "Preferred Reporting Items for Systematic Reviews and MetaAnalyses: The PRISMA Statement”. Open Med, 3(3); 123-130, 2009.

[33] Y. Sun, N. Wang, X. Guo, and Z. Peng, "Understanding the acceptance of mobile health services: a comparison and integration of alternative models," Journal of Electronic Commerce Research, vol. 14, no. 2, p. 183, 2013.

[34] J. P. Wisdom, M. A. Cavaleri, A. J. Onwuegbuzie, and C. A. Green, "Methodological reporting in qualitative, quantitative, and mixed methods health services research articles," Health services research, vol. 47, no. 2, pp. 721-745, 2012.

[35] X. Zhang, X. Guo, K.-h. Lai, F. Guo, and C. Li, "Understanding gender differences in m-health adoption: a modified theory of reasoned action model," Telemedicine and e-Health, vol. 20, no. 1, pp. 39-46, 2014.

[36] X. Zhang, X. Han, Y. Dang, F. Meng, X. Guo, and J. Lin, "User acceptance of mobile health services from users' perspectives: The role of self-efficacy and response-efficacy in technology acceptance," Informatics for Health and Social Care, vol. 42, no. 2, pp. 194-206, 2017. 